\title{
ALMOST FIXED POINT THEOREMS
}

\author{
ADAM IDZIK \\ (Communicated by John B. Conway)
}

\begin{abstract}
A closed cover version of Hazewinkel and van de Vel's theorem on the existence of $\gamma$-almost fixed points for set-valued functions and for real Hausdorff topological vector spaces is proved. Generalizations of well-known fixed point theorems of the Schauder-Tychonoff type are presented.
\end{abstract}

1. Introduction. In $[\mathrm{HV}]$ Hazewinkel and van de Vel proved the following $\gamma$-almost fixed point theorem, conjectured by de Groot for Euclidean spaces (see Chapter IV in [W]):

THEOREM 1.1 (THEOREM 9.7 IN [HV]). Let $\gamma$ be a finite convex open cover of a locally convex space $E$ and let $f: E \rightarrow E$ be a continuous function. Then there is $V \in \gamma$ such that $V \cap f(V) \neq \varnothing$.

This theorem is closely related with the following, more than a half of a century existing,

ProBlem 1.2 (Schauder's conjecture, Problem 54 in The Scottish Book, see [Gr]). Every continuous function, from a nonempty compact and convex set in a topological vector space into itself, has a fixed point.

During the last twenty years this old conjecture was intensively examined by many mathematicians (see references in [H3 and H4]). For sets in normed spaces it has been proved by Schauder $[\mathbf{S}]$ and for sets in locally convex spaces by Tychonoff [T]. Generalizations to upper semicontinuous (u.s.c.) functions defined on convex subsets of locally convex spaces were made by many authors, e.g. Browder [B], Fan [F], Glicksberg [G], Himmelberg [H], Reich [R1]. In his paper, Matusov [M] gave a solution of Schauder's conjecture, but his proof was incorrect. Zima [Z] extended the fixed point theorem of Schauder to paranormed spaces (not necessarily locally convex). Afterwards, Rzepecki $[\mathbf{R}]$ and Hadžić $[\mathbf{H 1}, \mathbf{H 2}]$ obtained more general theorems.

In this paper we generalize Hazewinkel and van de Vel's theorem to u.s.c. functions defined on almost convex subsets of real Hausdorff topological vector spaces. As an application of this generalization we extend some theorems of Hadžić [H2], Himmelberg [H], Idzik [I1, I2], Rzepecki [R] and Sehgal and Morrison [SM] (in the paper [I2] we consider the case of single-valued functions). Unfortunately we do not solve Schauder's conjecture in general.

For other aspects of almost fixed point theory see [R2 and V].

Received by the editors February 11, 1987 and, in revised form, November 2, 1987.

1980 Mathematics Subject Classification (1985 Revision). Primary 47H10, 54H25; Secondary 52A07, 55M20.

Key words and phrases. Fixed point, convexoid cover, Cech homology functor, almost convex set. 
2. Preliminaries. Throughout this paper $E$ denotes a real Hausdorff topological vector space. For a topological space $X$ let $2^{X}$ denote the space of closed subsets of $X$ endowed with the exponential topology. Let $X$ and $Y$ be topological spaces.

By a function $\varphi: X \rightarrow 2^{Y}$ we always understand that for each $x \in X, \varphi(x)$ is a nonempty set. If $A \subset X$, then $\varphi(A)=\bigcup\{\varphi(x) \mid x \in A\}$ and if $B \subset Y$, $\varphi^{-1}(B)=\{x \in X \mid \varphi(x) \cap B \neq \varnothing\}$. A function $\varphi: X \rightarrow 2^{Y}$ is u.s.c. iff for each closed set $B \subset Y \varphi^{-1}(B)$ is a closed subset of $X$. The function $\varphi$ is point convex (point compact) iff for each $x \in X, \varphi(x)$ is convex (compact) subset of $Y$. A graph of $\varphi$ is a set $\Gamma_{\varphi}=\{(x, y) \mid y \in \varphi(x)\} \subset X \times Y$. The cardinality of a set $I$ is denoted by $|I|$.

We need two lemmas and two definitions.

LEMMA 2.1 (SEE E.G. [I1, LEMMA 4]). Let $X$ be a topological space, $Y$ a compact regular space and $\varphi: X \rightarrow 2^{Y}$. Then $\varphi$ is u.s.c. iff the graph $\Gamma_{\varphi}$ of $\varphi$ is closed in $X \times Y$.

LEMMA 2.2 (SEE [L, THEOREM 12]). Let $\gamma=\left\{V_{i} \mid i \in I\right\}$ be a finite $(|I|<$ $\aleph_{0}$ ) family of nonempty compact, convexoid subsets of $E, V=\bigcup\left\{V_{i} \mid i \in I\right\}$ ( $\gamma$ is a convexoid cover of $V$ ) and $N$ be a nerve complex of the cover $\gamma$. Then $H(V)=$ $H(N)$, where $H$ is the Cech homology functor defined on the category of compact Hausdorff spaces.

Definition 2.3 (SEE [HV AND I2]). Let $X$ be a subset of $E, \varphi: X \rightarrow 2^{X}$ be a function and a family $\gamma=\left\{V_{i} \mid V_{i} \subset E, i \in I\right\}$ cover the set $\varphi(X)$, i.e. $\varphi(X) \subset$ $\bigcup\left\{V_{i} \mid i \in I\right\}$. We say that $\varphi$ has $\gamma$-almost fixed point if there is $V \in \gamma$ such that $V \cap \varphi(V \cap X) \neq \varnothing$.

DEFINITION 2.4 (SEE $[\mathbf{H}]$ ). A subset $A \subset E$ is almost convex if for any neighborhood $V$ of $0 \in E$, and for any finite set $\left\{w_{1}, \ldots, w_{n}\right\} \subset A$ there exists a finite set $\left\{z_{1}, \ldots, z_{n}\right\} \subset A$ such that for each $i \in\{1, \ldots, n\}, z_{i}-w_{i} \in V$ and $\operatorname{co}\left\{z_{1}, \ldots, z_{n}\right\} \subset A$.

Proposition 2.5. If $A \subset E$ is an almost convex set, then $A$ is the dense subset of $\operatorname{co} A$.

COROLLARY 2.6. The closure of an almost convex set is a convex set.

3. A generalization of Hazewinkel and van de Vel's Theorem. The theorem below generalizes Theorem 1.2 in [12] (which in turn is an extension of Theorem 9.7 in $[\mathbf{H V}])$ to u.s.c. functions.

THEOREM 3.1. Let $D, C$ be nonempty almost convex sets in $E$ and $C$ a dense subset of $D$. Let $\varphi: D \rightarrow 2^{D}$ be an u.s.c. function such that $\varphi(x)$ is convex for all $x \in C$. If $\gamma=\left\{V_{i} \mid i \in I\right\}$ is a finite family covering $\varphi(D)$ and $V_{i}$ is a convex, closed subset of $D(i \in I)$, then $\varphi$ has $\gamma$-almost fixed point.

Proof. Let us define $\alpha=\left\{L \subset I \mid \cap\left\{V_{i} \mid i \in L\right\} \neq \varnothing\right\}$ and $\beta=\{J \mid J \in \alpha$ and for $\left.k \in I, J \cap\left\{V_{i} \mid i \in J \cup\{k\}\right\}=\varnothing\right\}$. We have $\beta \subset \alpha$. For every $J \in \beta$ we choose $p_{J}^{0} \in \bigcap\left\{V_{i} \mid i \in J\right\} \subset D$. Of course for $J_{1}, J_{2} \in \beta$ and $J_{1} \neq J_{2}$ we have $p_{J_{1}}^{0} \neq p_{J_{2}}^{0}$. For a neighbourhood $V$ of $0 \in E$, by our assumption, there are points $\left\{p_{J}^{V} \mid J \in \beta\right\} \subset C$ such that $p_{J}^{0}-p_{J}^{V} \in V$ for $J \in \beta$ and $\operatorname{co}\left\{p_{J}^{V} \mid J \in \beta\right\} \subset C$. Let 
us consider an injection $h^{r}(r=0$ or $V)$ from $|\beta|-1$ dimensional symplex $S$ with vertices $\{p L \mid L \in \beta\}$ to $\operatorname{co}\left\{p_{L}^{r} \mid L \in \beta\right\}$ defined by $h^{r}(p L)=p_{L}^{r}$ and then linearly. The function $h^{r}$ is continuous. For $L \in \alpha$, let $s_{L}$ denote the barycenter of the set $\{p J \mid J \in \beta, L \subset J\}$, i.e. $s_{L}=1 /|\{J \mid J \in \beta, L \subset J\}| \sum_{J \in \beta, L \subset J} p J$. Observe that $s_{L}=p L$ for $L \in \beta$. For each $i \in I, L \in \beta$ and $i \in L$ there exist $k \in I(k=|L|)$ and $L_{j}^{i} \in \alpha(j=1, \ldots, k)$ such that $L_{1}^{i}=\{i\}, L_{k}^{i}=L$ and $L_{j-1}^{i} \subset L_{j}^{i},\left|L_{j}^{i}\right|-\left|L_{j-1}^{i}\right|=1$ $(j=2, \ldots, k)$. There may exist many such sequences $s(i, L)=\left\{L_{1}^{i}, \ldots, L_{k}^{i}\right\}$ joining $\{i\}$ and $L$ in this way. Let

$$
S_{i}=\bigcup\left\{\operatorname{co}\left\{s_{L_{1}^{i}}, \ldots, s_{L_{k}^{i}}\right\} \mid s(i, L)=\left\{L_{1}^{i}, \ldots, L_{k}^{i}\right\}, L \in \beta\right\}=\operatorname{co}\{p J \mid i \in J \in \beta\} .
$$

We have $h^{0}\left(S_{i}\right) \subset V_{i}$. Let us define a function $\psi_{V}: S \rightarrow 2^{S}$ as follows: For $x \in S$, $h^{V}(x) \in C$ and $\varphi\left(h^{V}(x)\right)=\bigcup\left\{V_{i}^{x} \mid i \in I_{V}^{x}\right\}$, where

$$
I_{V}^{x}=\left\{i \in I \mid V_{i}^{x}:=V_{i} \cap \varphi\left(h^{V}(x)\right) \neq \varnothing\right\} .
$$

Now, let us define

$$
\alpha_{V}^{x}=\left\{L \subset I_{V}^{x} \mid \bigcap\left\{V_{i}^{x} \mid i \in L\right\} \neq \varnothing\right\}
$$

and

$$
\beta_{V}^{x}=\left\{J \mid J \in \alpha_{V}^{x} \text { and for } k \in I \backslash J, \bigcap\left\{V_{i}^{x} \mid i \in J \cup\{k\}\right\}=\varnothing\right\} .
$$

We have $\beta_{V}^{x} \subset \alpha_{V}^{x}, \alpha_{V}^{x} \subset \alpha$ and for $L^{x} \in \beta_{V}^{x}$ there exists $L \in \beta$ such that $L^{x} \subset L$. Similarly we can choose $p_{J}^{x} \in \bigcap\left\{V_{i}^{x} \mid i \in J\right\}$ for $J \in \beta_{V}^{x}$. Observe that the nerve complex of the cover $\left\{V_{i}^{x} \mid i \in I_{V}^{x}\right\}$ of $\varphi\left(h^{V}(x)\right)$ is the same as that of the nerve complex of the cover $\left\{\operatorname{co}\left\{p_{J}^{x} \mid J \in \beta_{V}^{x}\right\} \cap V_{i}^{x} \mid i \in I_{V}^{x}\right\}$ of $\operatorname{co}\left\{p_{J}^{x} \mid J \in \beta_{V}^{x}\right\}$, and it is acyclic by Lemma 2.2. For each $x \in S$ and $i \in I_{V}^{x}$ we can also define

$$
\begin{aligned}
& S_{i}^{x}=\bigcup\left\{\operatorname{co}\left\{s_{L_{1}^{i}}, \ldots, s_{L_{k}^{i}}\right\} \mid s(i, L)=\left\{L_{1}^{i}, \ldots, L_{k}^{i}\right\}, L^{x} \in \beta_{V}^{x}, L_{1}^{i}=\{i\},\right. \\
& \left.L_{k}^{i}=L^{x} \text { and } L_{j-1}^{i} \subset L_{j}^{i},\left|L_{j}^{i}\right|-\left|L_{j-1}^{i}\right|=1, j=2, \ldots, k\right\} .
\end{aligned}
$$

The set $S_{i}^{x}$ is acyclic (it is contractible to $\left.s_{\{i\}}\right), S_{i}^{x} \subset S_{i}$ and for $i_{1} \in I_{V}^{x}(l=$ $1, \ldots, n)$ the set

$$
\begin{gathered}
S_{i_{1}}^{x} \cap \cdots \cap S_{i_{n}}^{x}=\bigcup\left\{\operatorname{co}\left\{s_{L_{1}^{i}}, \ldots, s_{L_{k}^{i}}\right\} \mid i=\left\{i_{1}, \ldots, i_{n}\right\}, s(i, L)=\left\{L_{1}^{i}, \ldots, L_{k}^{i}\right\},\right. \\
L^{x} \in \beta_{V}^{x}, L_{1}^{i}=i, L_{k}^{i}=L^{x} \text { and } L_{j-1}^{i} \subset L_{j}^{i}, \\
\left.\quad\left|L_{j}^{i}\right|-\left|L_{j-1}^{i}\right|=1, j=2, \ldots, k\right\},
\end{gathered}
$$

if nonempty, is also acyclic (it is contractible to $s_{L_{1}^{i}}$ ). From the last formula it follows that the cover $\left\{S_{i}^{x} \mid i \in I_{V}^{x}\right\}$ is convexoid and its nerve complex is the same as the nerve complex of the cover $\left\{V_{i}^{x} \mid i \in I_{V}^{x}\right\}$ and thus acyclic. We put $\psi_{V}(x)=$ $\bigcup\left\{S_{i}^{x} \mid i \in I_{V}^{x}\right\}$. By Lemma 2.2, $\psi_{V}(x)$ is acyclic for $x \in S$.

Because $h^{V}$ is continuous and $\varphi$ is u.s.c., then for $x_{n} \rightarrow x_{0}, I_{V}^{x_{n}} \subset I_{V}^{x_{0}}$ and $\beta_{V}^{x_{n}}$ is a refinement of $\beta_{V}^{x_{0}}$ for $n>n_{0}$ and some $n_{0}$. Thus for $n>n_{0}, i \in I_{V}^{x_{n}}$ implies $S_{i}^{x_{n}} \subset S_{i}^{x_{0}}$ and, consequently, $\psi_{V}\left(x_{n}\right) \subset \psi_{V}\left(x_{0}\right)$. By Lemma 2.1 it follows that $\psi_{V}$ is u.s.c.

From the Eilenberg-Montgomery fixed point theorem [EM] there exists $x_{V} \in$ $\psi_{V}\left(x_{V}\right)$. Let $x_{V} \in S_{i}^{x_{V}}$ for some $i \in I_{V}^{x_{V}}$. We have $h^{0}\left(S_{i}^{x_{V}}\right) \subset h^{0}\left(S_{i}\right) \subset V_{i}$ and $V_{i} \cap \varphi\left(h^{V}\left(x_{V}\right)\right) \neq \varnothing$. Let $\delta=\{V \mid V$ is a neighbourhood of $0 \in E\}$ and 
let $x$ be a cluster point of a subnet $\left\{x_{V^{\prime}}\right\}_{V^{\prime} \in \delta^{\prime}}$ of the net $\left\{x_{V}\right\}_{V \in \delta}$. Because $I$ is finite we can choose $i \in I$ and assume that $x_{V^{\prime}} \in S_{i}^{x_{V^{\prime}}}, h^{0}\left(S_{i}^{x_{V^{\prime}}}\right) \subset V_{i}$, and $V_{i} \cap \varphi\left(h^{V^{\prime}}\left(x_{V^{\prime}}\right)\right) \neq \varnothing$ for $V^{\prime} \subset V^{0}$ and some $V^{0} \in \delta^{\prime}$. Let us observe that $h^{V^{\prime}}\left(x_{V^{\prime}}\right)$ tends to $h^{0}(x) \in h^{0}\left(S_{i}\right) \subset V_{i}$ and by u.s.c. of $\varphi$ the set $\left\{y \mid V_{i} \cap \varphi(y) \neq \varnothing\right\}$ is closed. Thus $V_{i} \cap \varphi\left(h^{0}(x)\right) \neq \varnothing$ and because $h^{0}(x) \in V_{i}$ we have $V_{i} \cap \varphi\left(V_{i}\right) \neq \varnothing$.

Theorem 3.1 is no longer true when convex sets of the cover $\gamma$ are not contained in $D$. We give

EXAMPLE 3.2. In the space $E=R^{3}$, let us consider $p_{0}=(0,0,1), p_{1}=(-1,0,0)$, $p_{2}=(0,-1,0), p_{3}=(1,0,0), p_{4}=(0,1,0), q_{4 n}=(-1 /(4 n+1), 0,1-1 /(4 n+1))$, $q_{4 n+1}=(0,-1 /(4 n+2), 1-1 /(4 n+2)), q_{4 n+2}=(1 /(4 n+3), 0,1-1 /(4 n+3))$, $q_{4 n+3}=(0,1 /(4 n+4), 1-1 /(4 n+4))(n \in N)$ and the sets $F=\operatorname{int} \operatorname{co}\left\{p_{i} \mid i=\right.$ $0,1,2,3,4\}, G=\left\{r q_{m}+(1-r) q_{m+1} \mid 0 \leq r \leq 1, m \in N\right\}$. If $x=\left(x_{1}, x_{2}, x_{3}\right) \in F \cup G$, then there is only one $f(x)=\left(y_{1}, y_{2}, y_{3}\right) \in G$ with $y_{3}=x_{3}$ and the function $f: F \cup G \rightarrow G$ is well defined. Let us define also $g: G \rightarrow G$ by $g\left(q_{m}\right)=q_{m+2}$ $(m \in N)$ and then linearly on successive segments.

For $D=C=F \cup G, \gamma=\left\{V_{i} \mid i=1,2,3,4\right\}$, where $V_{i}=\operatorname{co}\left\{p_{i}, p_{i+1}, p_{0}\right\}$, $i=1,2,3,4\left(p_{5}=p_{1}\right)$ and the function $\varphi: D \rightarrow 2^{D}$ defined by $\varphi(x)=\{g(f(x))\}$ if $x \in D$; Theorem 3.1 is not valid.

4. Fixed point theorems. Now we give a few generalizations of fixed point theorems of Schauder-Tychonoff type.

DEFINITION 4.1 (CF. DEFINITION 2.2 IN [I2]). A set $B \subset E$ is convexly totally bounded (c.t.b. for short), if for every neighbourhood $V$ of $0 \in E$ there exist a finite subset $\left\{x_{i} \mid i \in I\right\} \subset B$ and a finite family of convex sets $\left\{C_{i} \mid i \in I\right\}$ such that $C_{i} \subset V$ for each $i \in I$ and $B \subset \bigcup\left\{x_{i}+C_{i} \mid i \in I\right\}$.

PROPOSITION 4.2. Every compact set in a locally convex space is a c.t.b. set.

THEOREM 4.3. Let $D, C$ be nonempty almost convex sets in $E$ and $C$ a dense subset of $D$. Let $\varphi: D \rightarrow 2^{D}$ be an u.s.c. function such that $\varphi(x)$ is convex for all $x \in C$. If $\overline{\varphi(D)}$ is a compact c.t.b. subset of $D$, then there exists $x \in D$ such that $x \in \varphi(x)$.

Proof. The closure of $\Gamma_{\varphi}$ in $E \times E$ defines a new function $\bar{\varphi}: \bar{D} \rightarrow 2^{D}$ which is u.s.c. by Lemma 2.1. Without loss of generality we may assume that the family $\delta=\{V \mid V$ is a neighbourhood of $0 \in E\}$ consist of closed symmetric and circled sets. For every $V \in \delta$, by Theorem 3.1 there exists a convex subset $S \subset V$ and $x \in \overline{\varphi(D)}$ such that for $S^{\prime}=(x+\bar{S}) \cap \bar{D}$ we have $S^{\prime} \cap \bar{\varphi}\left(S^{\prime}\right) \neq \varnothing$. It means that there exist $x_{V}, y_{V} \in \bar{D}$ such that $x_{V} \in \bar{\varphi}\left(y_{V}\right)$ and $x_{V}-y_{V} \in V+V$. Because $\overline{\varphi(D)}$ is compact, we can choose a subnet of $\left\{x_{V}\right\}_{V \in \delta}$ with a cluster point $x_{0} \in D$. But $\bar{\varphi}$ is u.s.c. thus $x_{0} \in \bar{\varphi}\left(x_{0}\right)=\varphi\left(x_{0}\right)$.

By Proposition 4.2, Theorem 4.3 generalizes Theorem 1 and Theorem 2 in $[\mathbf{H}]$.

Let $\delta$ be the fundamental system of neighbourhoods of 0 in $E$. We recall that a set $K \subset E$ is locally convex if for every $x \in K$ and every $V \in \delta$ there exists $U \in \delta$, such that $\operatorname{co}((x+U) \cap K) \subset x+V$. We say that $K \subset E$ is of $Z$ type (see [H3]) if for every $V \in \delta$ there exists $U \in \delta$ such that $\operatorname{co}(U \cap(K-K)) \subset V$.

PROPOSITION 4.4. In a locally convex space $E$ every subset $K \subset E$ is of $Z$ type and is a locally convex set. 
PROPOSITION 4.5. If $K \subset E$ is a compact set and $K$ is locally convex or $K$ is of $Z$ type, then it is c.t.b.

Proof. The first part of Proposition 4.5 is obvious. If $K$ is of $Z$ type, then for every $V \in \delta$ there exists $U \in \delta$ such that $\operatorname{co}(U \cap(K-K)) \subset V$. Because $K$ is compact, then there exists $\left\{x_{1}, \ldots, x_{n}\right\} \subset K$ such that $K \subset \bigcup_{i=1, \ldots, n}\left(x_{i}+U\right)$. Furthermore

$$
x_{i}+\operatorname{co}\left(U \cap\left(K-x_{i}\right)\right) \supset x_{i}+U \cap\left(K-x_{i}\right)=\left(x_{i}+U\right) \cap K \text { for } i=1, \ldots, n .
$$

Thus $\left\{x_{i}+\operatorname{co}\left(U \cap\left(K-x_{i}\right)\right) \mid i=1, \ldots, n\right\}$ is the cover of $K$.

In view of Proposition 4.5, Theorem 1 in [R], Theorem 1 and Theorem 2 in [H2] and Theorem 8 in [H3] follow from our Theorem 4.3.

The theorem below is Sehgal and Morrison's version of Theorem 4.3 (cf. [SM]).

THEOREM 4.6. Let $D, C$ be nonempty almost convex sets in $E, C$ a dense subset of $D$, and let $K$ be a compact subset of $D$. Moreover let $H$ be a topological space and $\varphi: D \rightarrow 2^{H}$ and $\psi: K \rightarrow 2^{H}$ be two u.s.c. functions. We shall assume that either $H$ is regular or $H$ is Hausdorff and $\psi$ is point compact. We shall assume also that

(i) $\varphi(x) \cap K \neq \varnothing$ for $x \in D$,

(ii) $\psi^{-1}(\varphi(x))$ is convex for $x \in C$,

(iii) $\overline{\psi^{-1}(\varphi(D))}$ is a c.t.b. set.

Under these assumptions there exists $x_{0} \in K$ such that $\varphi\left(x_{0}\right) \cap \psi\left(x_{0}\right) \neq \varnothing$.

Proof. The function $\hat{\varphi}: D \rightarrow 2^{K}$ is defined as follows: $\hat{\varphi}(x)=\psi^{-1}(\varphi(x))$ satisfies the assumptions of Theorem 4.3 and the proof proceeds in a similar way as the proof of Theorem 3 in [I1].

Comparing Theorem 4.3 and Schauder's conjecture we formulate

PROBLEM 4.7. Is every compact, convex set in a real Hausdorff topological vector space c.t.b.?

A positive answer to this question solves the problem of Schauder.

ACKNOWLEDGMENT. I am grateful to the referee for helpful comments.

\section{REFERENCES}

[B] F. E. Browder, The fixed point theory of multivalued mappings in topological vector spaces, Math. Ann. 177 (1968), 283-301.

[EM] S. Eilenberg and D. Montgomery, Fixed point theorems for multivalued transformations, Amer. J. Math. 58 (1946), 214-222.

[F] K. Fan, Fixed-point and minimax theorems in locally convex topological linear spaces, Proc. Nat. Acad. Sci. U.S.A. 38 (1952), 121-126.

[G] I. Glicksberg, A further generalization of the Kakutani fixed-point theorem, with application to Nash equilibrium points, Proc. Amer. Math. Soc. 3 (1952), 170-174.

[Gr] A. Granas, KKM-maps and their applications to nonlinear problems, The Scottish Book, Ed., R. D. Mauldin, Birkhäuser, Basel and Boston, Mass., 1981, pp. 45-61.

[H1] O. Hadžic, A fixed point theorem for the sum of two mappings, Proc. Amer. Math. Soc. 85 (1982), 37-41.

[H2] __, On Kakutani's fixed point theorem in topological vector spaces, Bull. Acad. Polon. Sci. Sér. Sci. Math. 30 (1982), 141-144.

[H3] _ Fixed point theorems in not necessarily locally convex topological vector spaces, Functional Analysis, Proc. Dubrovnik 1981, Lecture Notes in Math., vol. 948, Springer-Verlag, Berlin, Heidelberg, New York, 1982, pp. 118-130. 
[H4] _ Fixed point theory in topological vector spaces, University of Novi Sad, Novi Sad, 1984.

[HV] M. Hazewinkel and M. van de Vel, On almost-fixed-point theory, Canad. J. Math. 30 (1978), 673-699.

[H] C. J. Himmelberg, Fixed points of compact multifunctions, J. Math. Anal. Appl. 38 (1972), 205-207.

[I1] A. Idzik, Remarks on Himmelberg's fixed point theorems, Bull. Acad. Polon. Sci. Sér. Sci. Math. Astronom. Phys. 26 (1978), 909-912.

[I2] _ On $\gamma$-almost fixed point theorems. The single-valued case, Bull. Polish Acad. Sci. Math. 35 (1987), 461-464.

[L] J. Leray, Sur la forme des espaces topologiques et sur les points fixés des représentations, J. Math. Pures Appl. 24 (1945), 95-167.

[M] V. E. Matusov, Obobščenie teoremy o nepodvižnoi toðke Tihonova, Dokl. Akad. Nauk UzSSR 2 (1978), 12-14. (Russian)

[R1] S. Reich, Fixed points in locally convex spaces, Math. Z. 125 (1972), 17-31.

[R2] _ _ The almost fixed point property for nonexpansive mappings, Proc. Amer. Math. Soc. 88 (1983), 44-46.

[R] B. Rzepecki, Remarks on Schauder's fixed point principle and its applications, Bull. Acad. Polon. Sci. Sér. Sci. Math. 27 (1979), 473-480.

[S] J. Schauder, Der Fixpunktsatz in Funktionalräumen, Studia Math. 2 (1930), 171-180.

[SM] V. M. Sehgal and E. Morrison, A fixed point theorem for multifunctions, Proc. Amer. Math. Soc. 38 (1973), 643-646.

[T] A. Tychonoff, Ein Fixpunktsatz, Math. Ann. 111 (1935), 767-776.

[V] M. van de Vel, The intersection property: A contribution to almost fixed point theory, Thesis, Univ. Instelling Antwerpen, 1975.

[W] T. van der Walt, Fixed and almost fixed points, Thesis, Math. Centre, Amsterdam, 1963.

[Z] K. Zima, On Schauder's fixed point theorem with respect to para-normed space, Comment. Math. Prace Mat. 19 (1977), 421-423.

Institute of Computer Science, Polish Academy of Sciences, 00-901 WarsaW, PKIN, POLAND 